\title{
Identification of a four-microRNA panel in serum as promising biomarker for colorectal carcinoma detection
}

\author{
Guocheng Huang ${ }^{1,2}$, Benlin Wei ${ }^{1}$, Zebo Chen ${ }^{1}$, Jingyao Wang ${ }^{1}$, Liwen Zhao ${ }^{1,3}$, Xiqi \\ Peng ${ }^{1,2}$, Kaihao Liu'1,3, Yongqing Lai**,1,2 \& Liangchao Ni*,1,2 \\ ${ }^{1}$ Guangdong \& Shenzhen Key Laboratory of Male Reproductive Medicine \& Genetics, Peking University Shenzhen Hospital, \\ Shenzhen, Guangdong 518036, China \\ ${ }^{2}$ Shantou University Medical College, Shantou, Guangdong 515041, China \\ ${ }^{3}$ Anhui Medical University, Hefei, Anhui 230032, China \\ *Author for correspondence: Tel.: +86 075583923333 5862; Incord@163.com \\ **Author for correspondence: yqlord@163.com
}

Background: Screening for colorectal carcinoma (CRC) lacks an efficient, inexpensive and noninvasive approach. The stable presence of serum miRNA is expected to become a new diagnostic marker. Materials \& methods: Based on 135 CRC patients and 135 normal controls, this study was conducted in three phases to identify suitable serum miRNA for CRC diagnosis by using quantitative reverse transcription PCR. Bioinformatic assays were used for target genes prediction and functional annotation. Results: Serum expression level of seven miRNAs were significantly different between CRC patients and the normal controls. The final diagnostic panel (area under the curve $=0.893$; sensitivity $=81.25 \%$, specificity $=73.33 \%$ ) consists of miR-203a-3p, miR-145-5p, miR-375-3p and miR-200c-3p. Conclusion: The four-miRNA panel may serve as a novel, noninvasive biomarker for CRC diagnosis and screening.

First draft submitted: 23 December 2019; Accepted for publication: 9 April 2020; Published online: 16 July 2020

Keywords: biomarker • colorectal cancer • microRNA • miR-145-5p • miR-200c-3p • miR-203a-3p • miR-375-3p

Colorectal carcinoma (CRC) is one of the top three common cancers worldwide and was responsible for an estimated 145,600 new cases and 51,020 deaths in the USA in 2019 [1]. Surgical excision of the primary neoplasm with relevant lymph nodes is the main treatment method for limited and regional CRC, whereas chemotherapy is used for unresectable tumor and the prevention of recurrence [2]. The relative 5-year survival rates of patients with limited and regional CRC are 90 and 71\%, respectively, whereas survival rate for those with distant disease is $14 \%$, which indicates that early diagnosis has a great impact on survival rate [1]. Currently, several methods such as fecal occult blood test, colonoscopy and imaging techniques, including MRI and CT, are available to screen and diagnose CRC. However, colonoscopy is an invasive technique and requires full bowel preparation and sedation, which may result in low compliance [3]. CT and MRI, by contrast, are noninvasive but do not optimize cost and reliability. Fecal occult blood test and carcinoembryonic antigen blood test are economical but have low sensitivity and specificity for diagnosis of CRC [4]. Thus, it is important to identify new potential markers with high sensitivity and specificity for the early detection of CRC.

MicroRNAs (miRNAs) are endogenous noncoding small RNAs that regulate gene expression by combining with the $3^{\prime}$ untranslated region of related mRNAs, causing mRNA degradation or translational inhibition [5]. MicroRNAs have been found to participate in the development of many types of malignant tumors and could function as diagnostic biomarkers. In the past several years, many studies have revealed that circulating miRNAs are highly stable by being mainly contained in lipid or lipoprotein complexes, such as microvesicles, exosomes or apoptotic bodies [6-8]. Therefore, certain blood microRNAs could serve as potential noninvasive diagnosis markers of CRC.

In this study, we assessed possible serum biomarkers by investigating expression profiles of microRNA of CRC patients based on quantitative reverse transcription PCR (RT-qPCR). The study was conducted in three phases to 


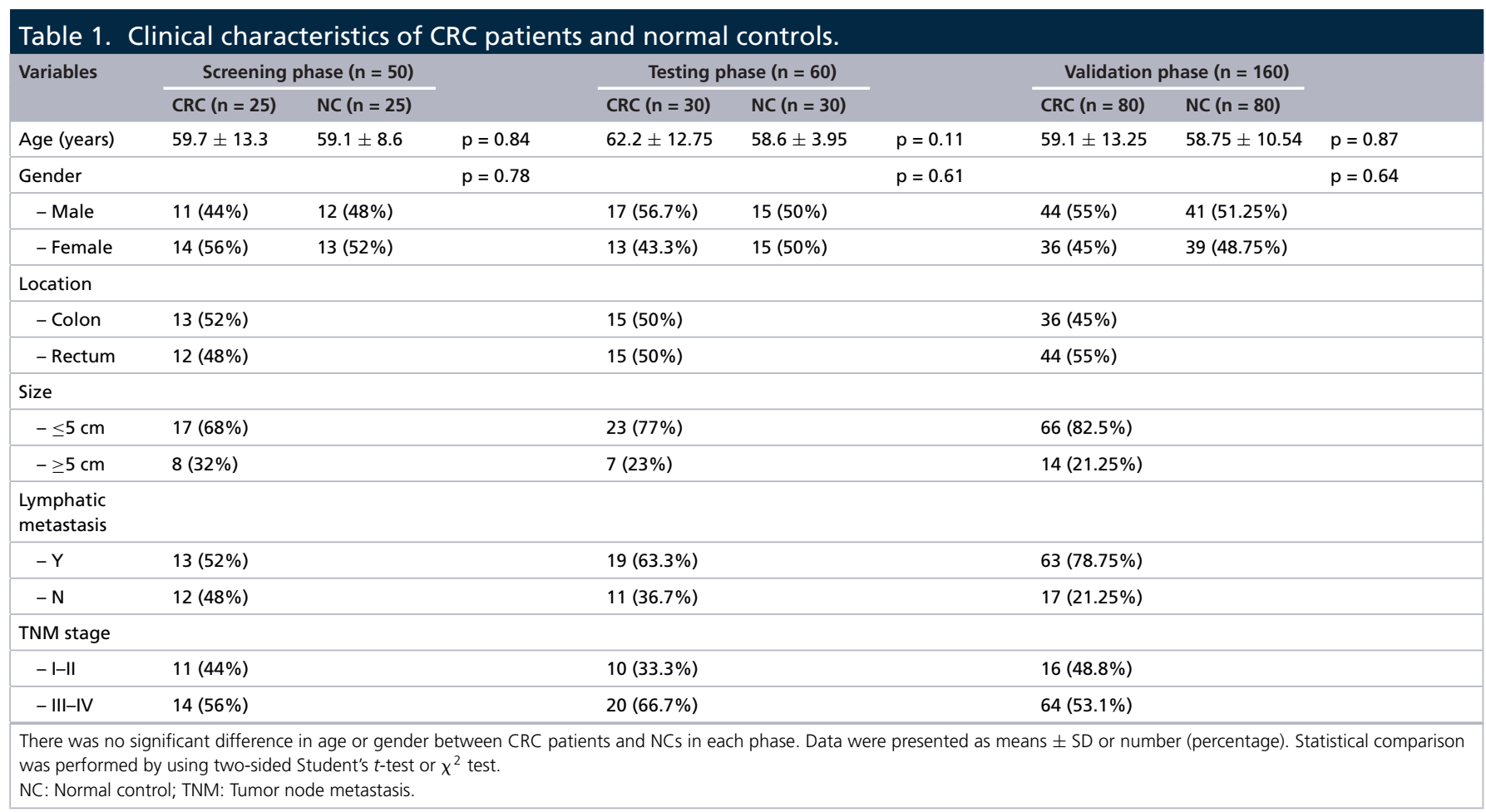

identify key microRNAs with good diagnostic value. First, 29 serum miRNAs were screened with five CRC pools and five normal controls (NCs) pools. Then, significantly expressed serum miRNAs were further validated twice in the testing phase and the validation phase. Finally, these candidate miRNAs were analyzed with backward stepwise logistic regression for construction of a high-efficiency diagnostic panel. Furthermore, correlation between the serum miRNAs relative expression and clinical manifestations were investigated. Additionally, potential biological functions of these miRNAs were explored by using bioinformatic analysis.

\section{Materials \& methods}

\section{Collection of human serum sample}

This study was reviewed and approved by the Ethics Committee of Shenzhen Hospital, Peking University. Each participating patient fully understood and signed an informed consent form. We collected serum samples from 135 histopathologically confirmed colorectal adenocarcinoma (CRC) patients and 135 NCs from January 2016 to May 2019 at Shenzhen Hospital, Peking University (Shenzhen, China). Pathological characteristics were determined according to the $\mathrm{WHO}$ criteria. The patients had been diagnosed with CRC before receiving any treatments. In NCs, we used serum samples from healthy volunteers with no cancer history, and no acute or chronic diseases. The clinical manifestations of the participants are shown in Table 1.

For the sample preparation, $10 \mathrm{ml}$ of peripheral blood was collected before any treatment and centrifuged at $3000 \times g$ for $10 \mathrm{~min}$ at $4^{\circ} \mathrm{C}$ within $2 \mathrm{~h}$. Then, the serum was stored in fresh tubes at $-80^{\circ} \mathrm{C}$ until RNA the experiment was conducted. Before RNA extraction, NanoDrop 2000c system (Thermo Fisher Scientific, MA, USA) was used to test hemolysis of all serum samples by measuring the absorbance of free hemoglobin at $414 \mathrm{~nm}$. Serum samples with $\mathrm{OD}_{414}$ greater than 0.2 were likely hemolytic and excluded from the study [9].

\section{Study design}

We conducted the study in three phases to identify serum miRNAs as potential CRC markers (Figure 1). A highefficiency diagnosis panel was constructed with these candidate miRNAs. Then, correlation between the serum miRNAs relative expression and clinical manifestations were investigated. Additionally, we also conducted target genes prediction, Kyoto Encyclopedia of Genes and Genomes (KEGG) pathway enrichment analysis and Gene Ontology (GO) functional annotation of these significantly expressed miRNAs. 


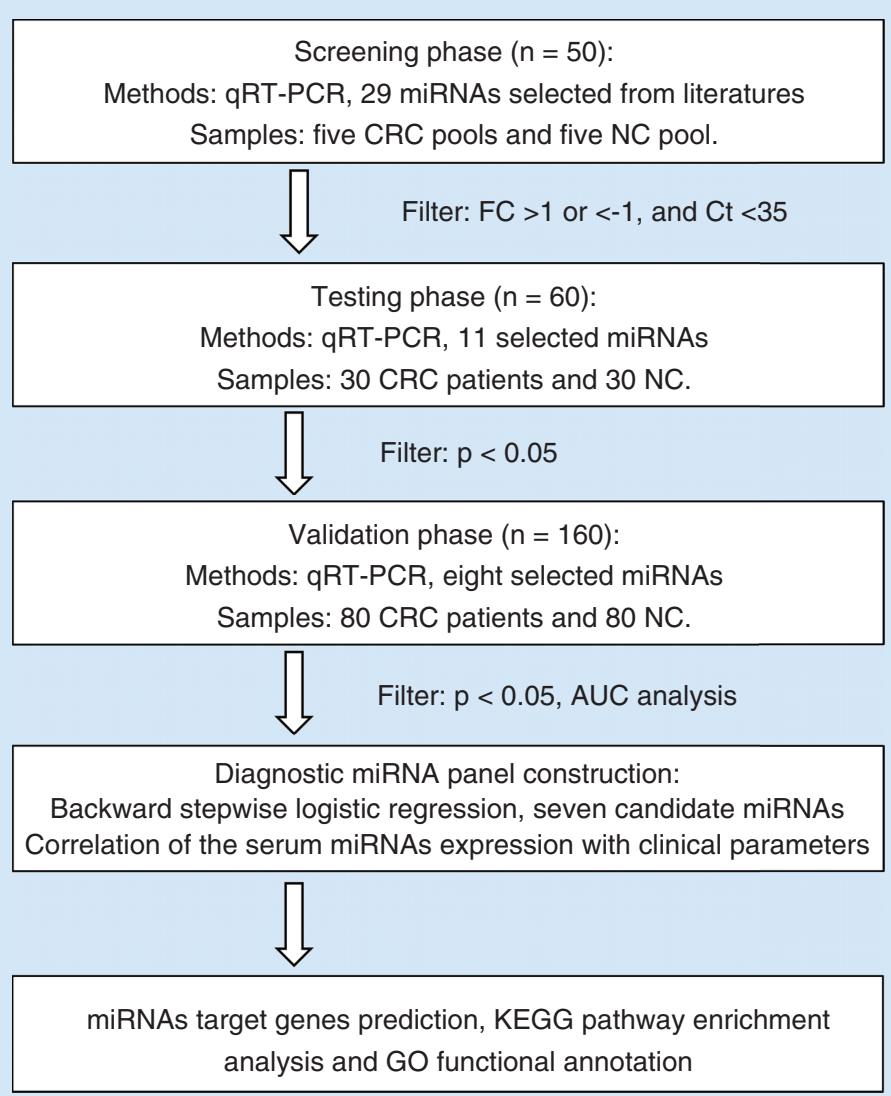

Figure 1. Overview of the study design.

AUC: Area under the curve; CRC: Colorectal carcinoma; Ct: Cycle threshold; FC: Fold change; GO: Gene ontology; KEGG: Kyoto Encyclopedia of Genes and Genomes; NC: Normal control; PCR: Polymerase chain reaction.

Screening phase: by searching in PubMed with keywords '(colon OR rectum OR colorectal) AND (miRNA OR microRNA)', 29 miRNAs (miR-143-5p, miR-200c-3p, miR-106a-5p, miR-200c-5p, miR-106b-5p, miR-3753p, miR-145-5p, miR-298, miR-10a-5p, miR-145-3p, miR-203a-3p, miR-215-5p, miR-877-5p, miR-6852-3p, miR-342-5p, miR-200a-3p, miR-4709-5p, miR-137-3p, miR-34a-3p, miR-585-3p, miR-122-3p, miR-193a-3p, miR-133b, miR-885-3p, miR-183-3p, miR-125b-5p, miR-92b-5p, miR-361-5p and miR-139-5p) that have been revealed to be closely related to colon or rectum cancer cells/tissues for which there are not enough plasma or serum expression studies were included in the experiment. Then, with five samples pooled as one pool, we pooled serum samples from 25 CRC patients and $25 \mathrm{NCs}$ into five CRC pools and five NC pools. These five CRC pools samples and five NCs pools were used to screening serum detectable miRNA among these 29 miRNAs. miRNAs with, single-peaked melting curve, fold change $(\mathrm{FC})>1$ or $<-1$ and cycle threshold $(\mathrm{Ct})<35$ were chosen for testing phase.

Testing phase: to verify the serum expression levels of these selected miRNAs, another 30 CRC patients and 30 NCs were used for further testing. miRNAs with $\mathrm{p}<0.05$ were identified as candidate miRNAs for further evaluation.

Validation phase: the candidate miRNAs identified in the previous two phases were further tested with 80 CRC patients and $80 \mathrm{NCs}$. In this phase, the p-value of each candidate miRNAs was calculated for verification of differential expression. The receiver operating characteristic (ROC) curve was drawn and the area under the curve (AUC) was used for evaluating the diagnostic capability. Then, the diagnostic panel was constructed by these candidate miRNAs based on the logistic regression analysis. 
Bioinformatic analysis: miRWalk 3 (http://mirwalk.umm.uni-heidelberg.de/) was used for target genes prediction. Enrichr database (http://amp.pharm.mssm.edu/Enrichr/) was used for KEGG pathway enrichment analysis and GO functional annotation.

\section{RNA extraction \& RT-qPCR}

Total RNA was extracted from the serum samples by using TRIzol ${ }^{\text {TM }}$ LS Reagent (Invitrogen, CA, USA), and RNA concentrations were determined using a NanoDrop 2000c system (Thermo Fisher Scientific). Then cDNA was prepared by reverse transcription of RNA using the PrimeScript RT reagent Kit (TaKaRa, Dalian, China). Later, quantitative real-time PCR (qPCR) was performed to detect the expression level of these miRNAs using the SYBR Pre-mix Ex Taq II (TaKaRa, Dalian, China) on LightCycler 480 Real-Time PCR System (Roche Diagnostics, Mannheim, Germany). The qPCR reaction was performed for $20 \mathrm{~s}$ at $95^{\circ} \mathrm{C}$, then 40 cycles $95^{\circ} \mathrm{C}$ for $10 \mathrm{~s}, 60^{\circ} \mathrm{C}$ for $20 \mathrm{~s}$ and then $70^{\circ} \mathrm{C}$ for $10 \mathrm{~s}$. Two microliters of synthetic Caenorbabditis elegans miR-39 (cel-miR-39) (10 nM/l, RiboBio, Guangzhou, China) was added into each sample as an internal control to normalize efficiency variation between samples during RNA extraction, reverse transcription and qPCR processes. The $2^{-\Delta \Delta C q}$ method was used to analyze miRNA expression levels [10].

Statistical analysis

A $2^{-\Delta \Delta C q}$ method was used for FCs calculation of miRNAs. microRNA expression level was calculated by subtracting the Cq value of the endogenous control cel-miR-39-3p ( $\Delta \mathrm{Cq}$ ). The ROC curve and AUC were used to evaluate the sensitivity, specificity and diagnostic capacity of each miRNA. The diagnostic efficacy was evaluated as following: AUC is $0.5-0.7$ (low), 0.7-0.9 (medium) and 0.9-1.0 (high). Logical regression was used to identify the most efficient diagnostic panel with the highest sensitivity and specificity. GraphPad 5.0 software and SPSS software version 22.0 were used for statistical analyses. Difference between groups was detected by using Student's- $t$-test or one-way analysis of variance. Data were presented as means \pm SD. $p<0.05$ was considered statistically significant.

\section{Results}

Characteristics of study subjects

As shown in Figure 1, we conducted this study in three separate phases: the screening phase, the testing phase and the validation phase. In total, 135 CRC patients and 135 healthy controls were involved in these three phases. The statistics and clinical characteristics of the participants are listed in Table 1. There was no significant difference in gender and age distribution between CRC patients and NCs at each phase.

\section{Selection of miRNAs from the screening phase}

Twenty-nine miRNAs selected from the literature were tested with five CRC pools and five NCs pools (Figure 2). Seven miRNAs were unable to detect or $\mathrm{Ct}>35$, whereas 22 miRNAs were detected with a single-peaked melting curve, $\mathrm{Ct}<35$. Among these 22 miRNAs, 11 miRNAs with $\mathrm{FC}>1$ or $<-1$, were selected as candidate miRNAs for further verification in the testing phase. In particular, four miRNAs (miR-10a-5p, miR-145-3p, miR-215-5p and miR-203a-3p) have higher expression in the CRC patients compared with the NCs. On the contrary, expression levels of seven miRNAs (miR-106a-5p, miR-106b-5p, miR-143-5p, miR-145-5p, miR-200c-3p, miR-200c-5p and miR-375-3p) were lower in CRC patients than NCs.

\section{Identification of candidate miRNAs in the testing phase}

The 11 selected miRNAs were further tested with 30 CRC patients and 30 NCs by RT-qPCR. As shown in Figure 3, expression levels of these miRNAs were largely consistent with the screening phase. Eight miRNAs with significantly different expression between CRC patients and NCs were identified as candidate miRNAs. miR-145-3p and miR-203a-3p demonstrated significantly higher expression in the CRC patients than the NCs $(p<0.05)$, while expression of the other six miRNAs (miR-106a-5p, miR-143-5p, miR-145-5p, miR-200c-3p, miR-200c-5p and miR-375-3p) was significantly lower in CRC patients than in NCs $(p<0.05)$. However, no significant difference had been found in three miRNAs (miR-10a-5p, miR-106b-5p and miR-215-5p).

\section{Evaluation of candidate miRNAs in the validation phase}

The eight candidate miRNAs identified previously were further tested with another 80 CRC patients and 80 NCs by using RT-qPCR analysis. Seven miRNAs, except miR-145-3p, were confirmed with a significant difference between 


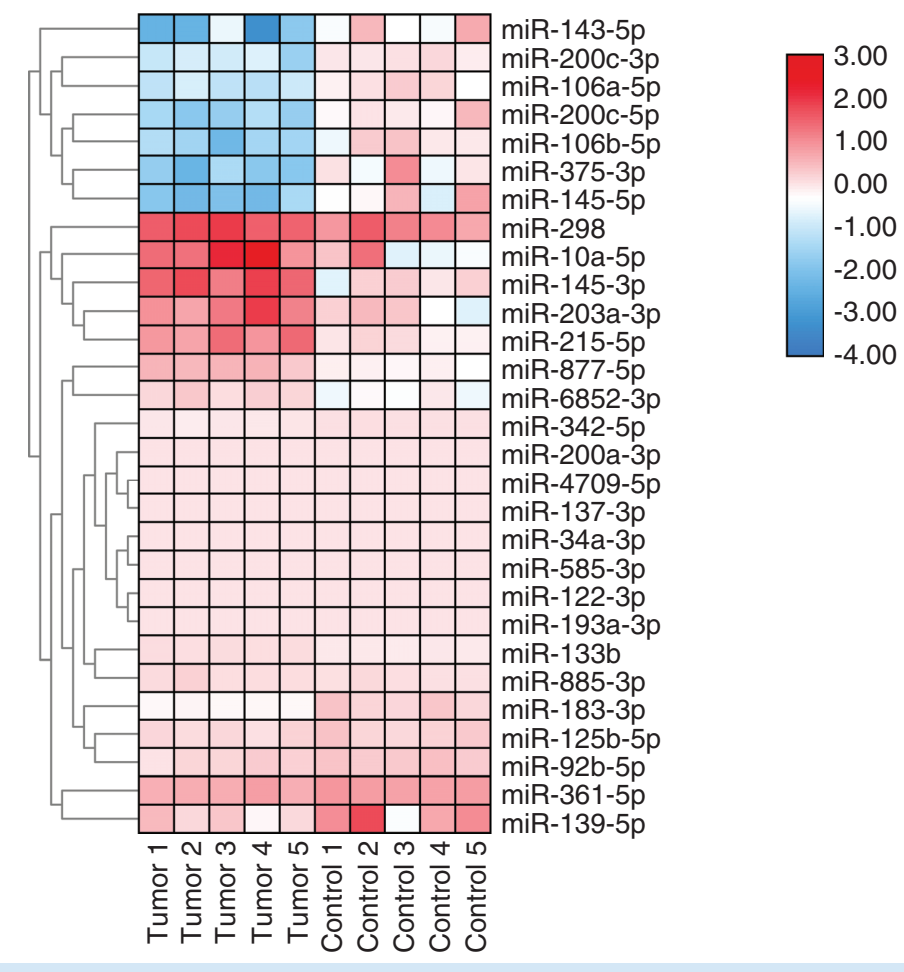

Figure 2. Heatmap of 29 miRNAs in the screening phase. Five CRC pools and five NCs pools were tested in this phase, and 11 miRNAs with FC $>1$ or $<-1$ were selected for further test.

FC: Fold change; NC: Normal control.

CRC patients and NCs (Figure 4). The relative expression level of miR-203a-3p was significantly increased in the serum of CRC patients. The other six miRNAs, including miR-106a-5p, miR-143-5p, miR-145-5p, miR-375-3p, miR-200c-3p and miR-200c-5p, were significantly downregulated.

ROC curve analyses were performed on each miRNA and AUCs for miR-203a-3p, miR-106b-5p, miR-143-5p, miR-145-5p, miR-375-3p, miR-200c-3p and miR-200c-5p were 0.712 (95\% CI: 0.633-0.791), 0.624 (95\% CI: 0.537-0.712), 0.745 (95\% CI: 0.669-0.821), 0.754 (95\% CI: 0.678-0.830), 0.715 (95\% CI: 0.637-0.793), 0.656 (95\% CI: 0.568-0.743) and 0.702 (95\% CI: 0.622-0.782), respectively (Figure 4).

miR-203a-3p, miR-143-5p, miR-145-5p, miR-375-3p and miR-200c-5p have moderate diagnostic ability while miR-106b-5p and miR-200c-3p have low diagnostic ability. To precisely diagnose CRC patients, backward stepwise logistic regression was used for a combination of the seven miRNAs. During this process, miR-143-5p, miR-200c$5 p$ and miR-106b-5p were removed and a four-miRNA panel (miR-203a-3p, miR-145-5p, miR-375-3p and miR-200c-3p) for diagnosis of CRC was constructed. ROC of the panel was drawn, and the AUC was 0.893 (95\% CI: $0.846-0.940$; sensitivity $=81.25 \%$, specificity $=73.33 \%$; Figure 5 ). The final logistic regression model was calculated with the equation: logit $(\mathrm{P})=0.474+2.376 \times$ miR-203a-3p-0.611 $\times$ miR-145-5p-2.711 $\times$ miR-375$3 \mathrm{p}-0.811 \times \mathrm{miR}-200 \mathrm{c}-3 \mathrm{p}$.

\section{Relationship between serum miRNAs expression \& clinical manifestations}

To further investigate the clinical significance of the four serum miRNAs (miR-203a-3p, miR-145-5p, miR-375-3p and miR-200c-3p), relationships between serum miRNAs expression levels and clinical parameters were analyzed by using Student's $t$-test or one-way ANOVA. When CRC patients in the testing phase and validation phase were grouped by tumor location, tumor size, lymphatic metastasis or pathology stage, there was no significant difference in the relative expression levels of miR-203a-3p, miR-145-5p and miR-200c-3p (Table 2). However, the relative expression level of miR-375-3p was downregulated in CRC patients with lymphatic metastasis or advanced pathology stage $(\mathrm{p}<0.001)$. 


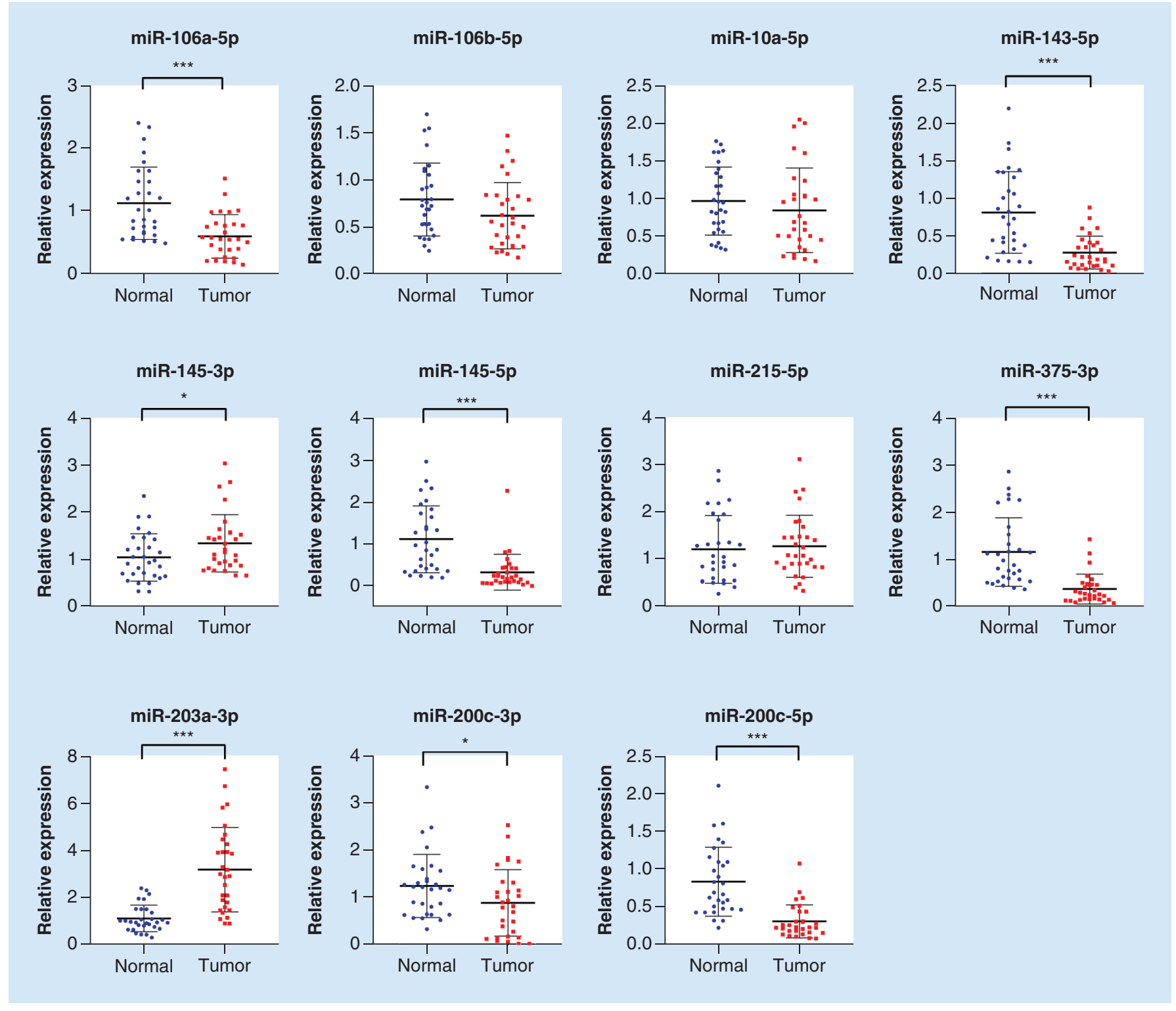

Figure 3. Eleven serum miRNAs expression level of 30 colorectal carcinoma patients and $\mathbf{3 0}$ normal controls in the testing phase. Eight miRNAs with significantly different expression between colorectal carcinoma patients and normal controls were identified as candidate miRNAs.

${ }^{*} \mathrm{p}<0.05 ;{ }^{* *} \mathrm{p}<0.01 ; * * * \mathrm{p}<0.001$.

Bioinformatic analysis of miR-203a-3p, miR-145-5p, miR-375-3p \& miR-200c-3p

Possible target genes of each miRNA were predicted by using the miRWalk 3. Genes predicted in more than two miRNAs were selected as target genes. A total of 625 genes were selected as target genes and the Enrichr database was used for KEGG pathway enrichment analysis and GO functional annotation. Sorted by p-value ranking, top ten $(\mathrm{p}<0.05)$ of the biological process, cellular component, molecular function and KEGG pathways were shown in Figure 6. Gene ontology functional annotation included positive regulation of MAP kinase activity (GO:0043406; $\mathrm{p}<0.001$ ), regulation of cytokine production involved in inflammatory response (GO:1900015; $\mathrm{p}<0.001$ ), positive regulation of gene expression (GO:0010628; $\mathrm{p}<0.001$ ), actin cytoskeleton (GO:0015629; $\mathrm{p}<0.01$ ), lytic vacuole (GO:0000323, $\mathrm{p}<0.01$ ), late endosome (GO:0005770; $\mathrm{p}<0.01$ ), 3',5'-cyclic-AMP phosphodiesterase activity (GO:0004115; p < 0.001), RNA polymerase II regulatory region sequence-specific DNA binding (GO:0000977; $\mathrm{p}<0.001)$ and $3^{\prime}, 5^{\prime}$-cyclic-nucleotide phosphodiesterase activity 


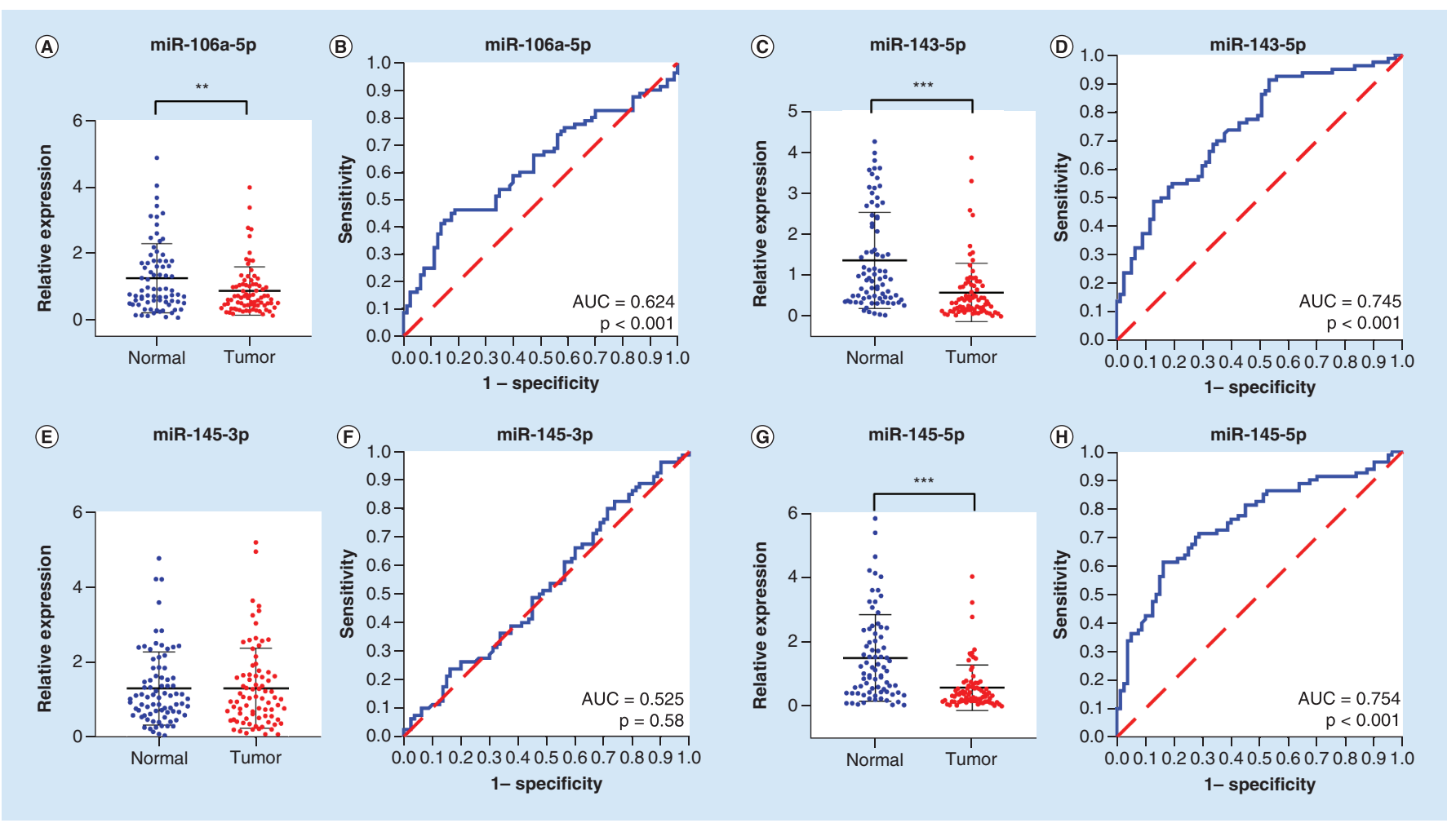

Figure 4. Serum expression level and receiver operating characteristic curve analyses of eight candidate miRNAs in validation phase. Eighty colorectal carcinoma patients and 80 normal controls were involved in this process.

${ }^{*} \mathrm{p}<0.05 ; * * \mathrm{p}<0.01 ; * * * \mathrm{p}<0.001$.

\begin{tabular}{|c|c|c|c|c|c|c|c|c|}
\hline Variables & miR-203a-3p & & miR-145-5p & & miR-375-3p & & miR-200c-3p & \\
\hline Location & & $p=0.77$ & & $p=0.89$ & & $p=0.41$ & & $p=0.54$ \\
\hline - Rectum & $2.14 \pm 1.59$ & & $0.56 \pm 0.47$ & & $0.64 \pm 0.39$ & & $1.17 \pm 1.41$ & \\
\hline Size & & $p=0.79$ & & $p=0.74$ & & $p=0.55$ & & $p=0.68$ \\
\hline$-\leq 5 \mathrm{~cm}$ & $2.10 \pm 1.85$ & & $0.57 \pm 0.68$ & & $0.68 \pm 0.40$ & & $1.04 \pm 1.35$ & \\
\hline $\begin{array}{l}\text { Lymphatic } \\
\text { metastasis }\end{array}$ & & $p=0.52$ & & $p=0.23$ & & $\mathrm{p}<0.001$ & & $p=0.19$ \\
\hline$-Y$ & $2.19 \pm 2.07$ & & $0.68 \pm 0.78$ & & $0.53 \pm 0.27$ & & $1.27 \pm 1.32$ & \\
\hline$-N$ & $1.94 \pm 1.36$ & & $0.49 \pm 0.62$ & & $0.86 \pm 0.43$ & & $0.89 \pm 1.21$ & \\
\hline TNM stage & & $p=0.48$ & & $p=0.42$ & & $\mathrm{p}<0.001$ & & $p=0.29$ \\
\hline
\end{tabular}

Colorectal carcinoma patients in the testing phase and the validation phase were grouped by tumor location, tumor size, lymphatic metastasis or pathology stages. Serum miR-375-3p relative expression was significantly related with lymphatic metastasis and TNM stage. Data were presented as means \pm SD. Statistical comparison was performed by using two-sided Student's $t$-test or $x^{2}$ test.

TNM: Tumor node metastasis.

(GO:0004114; $\mathrm{p}<0.001)$. Additionally, KEGG pathways included hypertrophic cardiomyopathy $(\mathrm{p}=0.005)$, colorectal carcinoma $(\mathrm{p}=0.005)$ and cell adhesion molecules $(\mathrm{p}=0.006)$. 


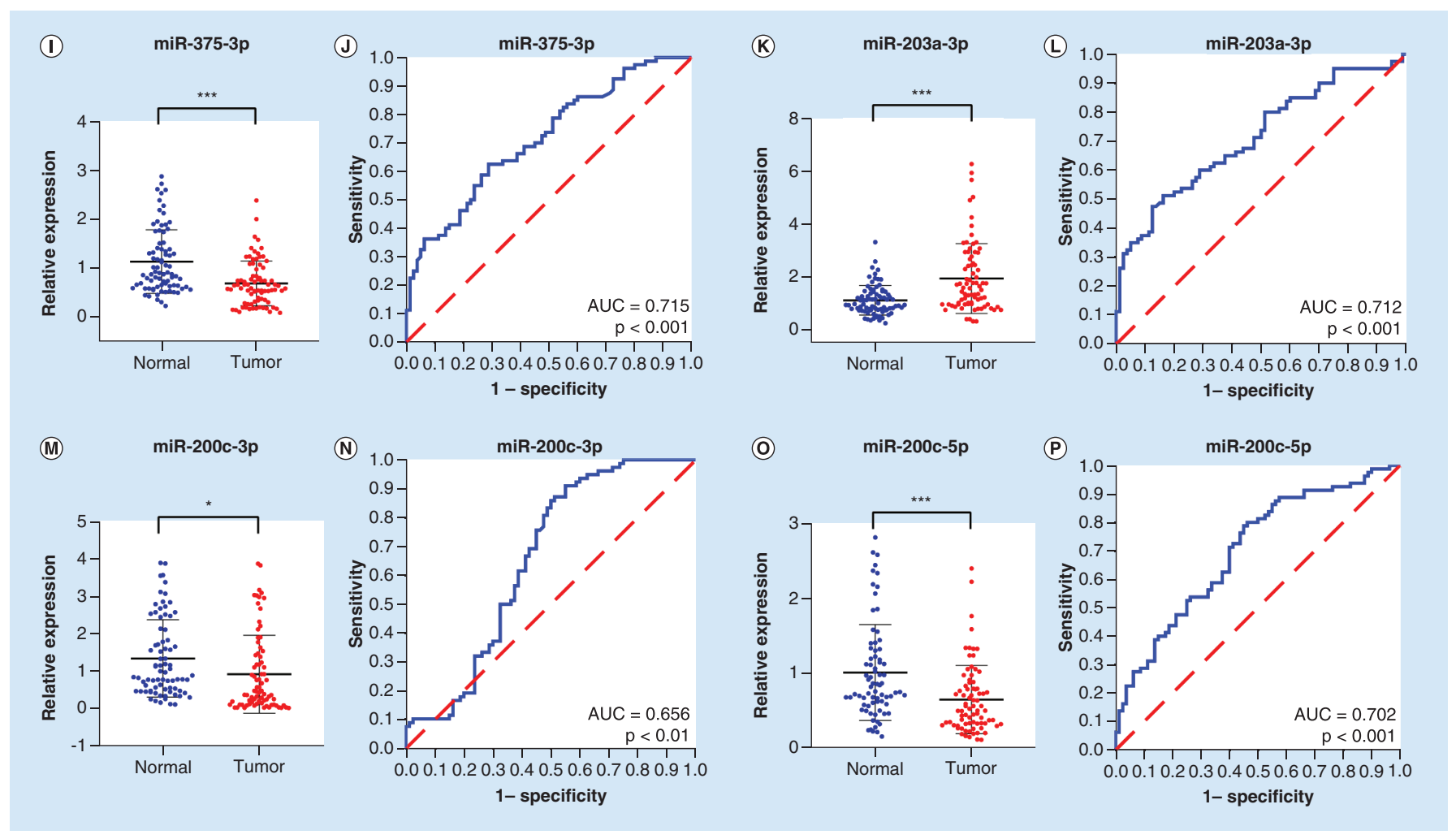

Figure 4. Serum expression level and receiver operating characteristic curve analyses of eight candidate miRNAs in validation phase (cont.). Eighty colorectal carcinoma patients and 80 normal controls were involved in this process.

${ }^{*} \mathrm{p}<0.05 ;{ }^{* *} \mathrm{p}<0.01 ; * * * \mathrm{p}<0.001$.

\section{Discussion}

Colorectal carcinoma (CRC) is the third most common cancer in the world, causing many deaths and a heavy financial burden each year. Early diagnosis and treatment can improve patients' survival rates and quality of life. Finding more sensitive diagnostic markers than those currently available promises to diagnose colorectal carcinoma earlier and improve patients' survival.

There is growing evidence that miRNA disorders are involved in the occurrence and progression of cancer including CRC. For instance, Gopalan et al. revealed that miR-498 was underregulated in CRC tissue and the proliferation capacity of miR-498 transfected CRC cells was reduced [11]. However, many studies focused on either single miRNA or CRC tissue expression profile. Due to the stable expression of miRNA in circulation, combination of several key blood miRNAs is expected to become a simple and reliable diagnostic marker for CRC.

In this study, the final diagnostic panel includes miR-203a-3p, miR-145-5p, miR-375-3p and miR-200c-3p. The relative expression level of miR-203a-3p was significantly increased in the serum of CRC patients. The other three miRNAs, including miR-145-5p, miR-375-3p and miR-200c-3p, were significantly downregulated. A small number of studies provided the expression level of these four miRNAs inside tissues or cells. miR-145-5p has been revealed to be underexpressed and exert tumor-suppressive effects in several kinds of cancer, including melanoma [12], bladder cancer [13], esophageal squamous cell carcinoma [14], as well as colorectal carcinoma [15]. Niu et al. suggested that miR-145-5p-targeted RHBDD1 and restrained its expression in CRC cells. Downregulation of miR-145-5p promoted tumorigenesis in CRC by upregulating RHBDD1, which causes overactivity of the EGFR-associated signaling pathway (EGFR/Raf/MEK/ERK cascades) [14]. Another miRNA, miR-375-3p, has been shown to inhibit the proliferation, invasion and migration of CRC cells by targeting SP1 and EMT-associated genes [16]. Mussnich et al. found that miR-375-3p contributed to cetuximab resistance in CRC patients [17].

MiR-200c-3p, an important member of the miR-200 family, reportedly acts as a tumor suppressor in CRC [18]. Srivastava $e t$ al. found that miR-200c-3p enriched in urine exosomes and could serve as a noninvasive biomarker for endometrial cancer [19]. Additionally, Chen et al. suggested that miR-203a-3p promoted CRC cell proliferation, 


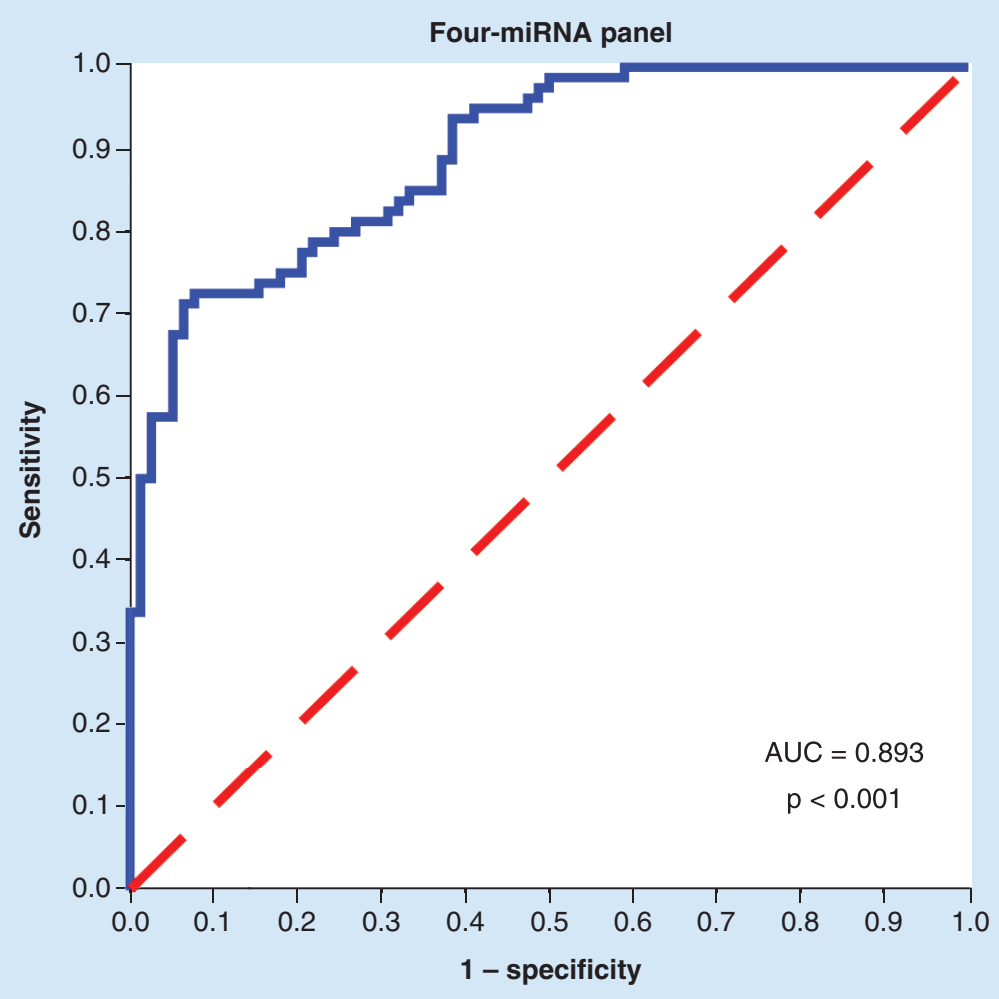

Figure 5. Receiver operating characteristic curve analyses of the four-miRNA panel. AUC the panel was 0.893 ( $95 \%$ Cl: $0.846-0.940$; sensitivity $=81.25 \%$, specificity $=73.33 \%$ ).

AUC: Area under the curve.

(A)

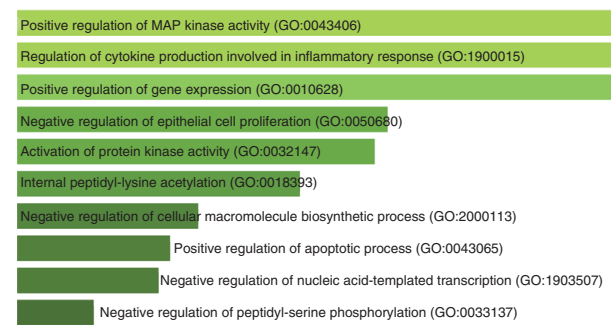

(C)

3', '5'-cyclic-AMP phosphodiesterase activity (GO:0004115)

RNA polymerase II regulatory region sequence-specific DNA binding (GO:0000977)

3', $5^{\prime}$-cyclic-nucleotide phosphodiesterase activity (GO:0004114)

Actin fliament binding (GO:0051015)

Transcriptional activator activity, RNA polymerase II core promoter proximal region sequence-specific binding (GO:0001077)

Transcriptional activator activity, RNA polymerase II transcription regulatory region sequence-specific binding (GO:0001228)

DNA binding (GO:0003677)

GTPase activator activity (GO:0005096)

Spectrin binding (GO:0030507)

ANA polymerase II transcription factor activity, sequence-specific transcription regulatory region DNA binding (GO:0001133)
(B)

Cellular component

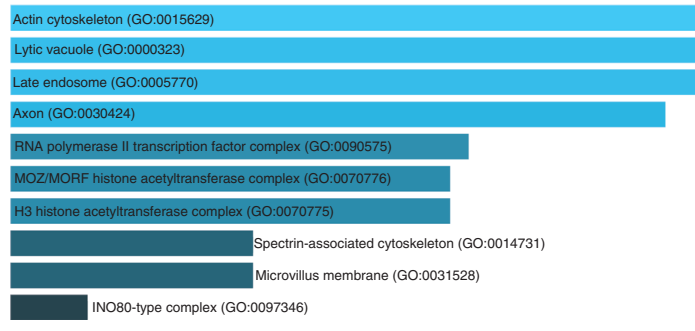

(D)

KEGG pathways

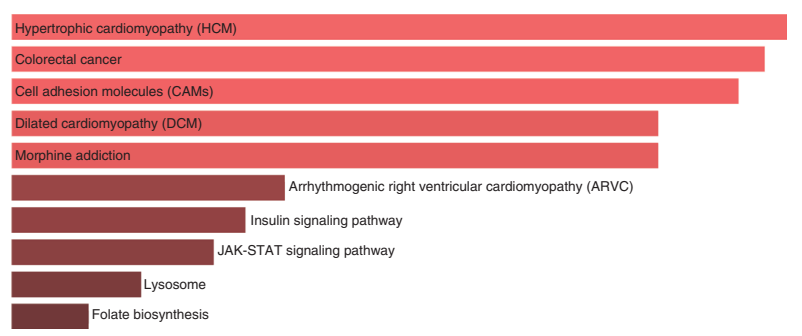

Figure 6. Gene ontology functional annotation and Kyoto Encyclopedia of Genes and Genomes pathway enrichment. (A) Biological process analysis; (B) cellular component analysis; (C) molecular function analysis; (D) KEGG pathway enrichment analysis. GO: Gene ontology; KEGG: Kyoto Encyclopedia of Genes and Genomes. 
colony formation, invasion, migration and inhibited apoptosis by suppressing the expression of PDE4D [20]. In this study, the serum expression level of miR-203a-3p was upregulated in the serum of CRC patients, which is consistent with the miR-203a-3p expression level of CRC tissue and cells in Chen's study. However, Qian et al. suggested that miR-203a-3p was downregulated in CRC tissues compared with adjacent normal tissues. miR-203a-3p served as a tumor suppressor of CRC cells by downregulating THBS2 expression [21]. Both studies used CRC cell lines SW480 and SW620, but their conclusions contradicted each other. Moreover, miR-203a-3p has been revealed as a tumor suppressor in gastric cancer [22], nasopharyngeal carcinoma [23] and non-small-cell lung cancer [24]. It is hard to say which study is more reliable. miRNA-203a-3p has different effects on various cancers, depending on the type of tumor and target gene. However, more studies have shown miR-203a-3p acted as a tumor suppressor rather than tumor promoter.

In this study, clinical manifestations of CRC patients including tumor location, tumor size, lymphatic metastasis and pathology stages were analyzed. There was no correlation between these clinical manifestations and relative expression levels of miR-203a-3p, miR-145-5p and miR-200c-3p. However, serum miR-375-3p expression levels in early CRC patients (stage I/II) were higher than serum miR-375-3p levels in patients with advanced CRC (stage III/IV). Therefore, we speculated that miR-375-3p was involved in the development and progression of colorectal carcinoma.

In short, the serum miRNAs expression level in this study was broadly consistent with that of CRC tissue or cell lines in previous studies. Detection of miR-203a-3p, miR-145-5p, miR-375-3p and miR-200c-3p expression levels in the serum could reflect their expression inside tissue and organs. Additionally, this study showed a significant difference in serum expression of miR-203a-3p, miR-145-5p, miR-375-3p and miR-200c-3p between CRC patients and NCs. The diagnostic panel that consists of these four miRNAs had good performance in the diagnosis of colorectal carcinoma than each one. Therefore, this study suggested that the panel of four serum miRNAs (miR-203a-3p, miR-145-5p, miR-375-3p and miR-200c-3p) could serve as a noninvasive biomarker for colorectal carcinoma.

\section{Future perspective}

In this study, we demonstrated that certain serum miRNAs are dysregulated in colorectal carcinoma. A combination of these miRNAs could further enhance their clinical application to serve as a noninvasive biomarker for screening of colorectal carcinoma.

\section{Summary points}

- Screening for colorectal carcinoma (CRC) lacks an efficient, inexpensive and noninvasive approach.

- The stable presence of serum miRNA is expected to become a new diagnostic marker.

- This study was conducted in three phases to assess expression of 29 serum miRNAs identified from the literature by using reverse transcription PCR.

- miR-203a-3p, miR-106a-5p, miR-143-5p, miR-145-5p, miR-200c-3p, miR-200c-5p and miR-375-3p were confirmed to show a significant difference between CRC patients and normal controls.

- A four-miRNA panel (miR-203a-3p, miR-145-5p, miR-375-3p and miR-200c-3p) for diagnosis of CRC was constructed (area under the curve $=0.893,95 \% \mathrm{Cl}$ : $0.846-0.940$; sensitivity $=81.25 \%$, specificity $=73.33 \%$ ).

- Bioinformatic analysis results of miR-203a-3p, miR-145-5p, miR-375-3p and miR-200c-3p were involved in positive regulation of MAP kinase activity $(\mathrm{GO}: 0043406, \mathrm{p}<0.001)$, regulation of cytokine production involved in inflammatory response (GO:1900015, p $<0.001)$, late endosome (GO:0005770, $p<0.01)$, RNA polymerase II regulatory region sequence-specific DNA binding (GO:0000977, $p<0.001)$ and pathways of colorectal carcinoma $(p=0.005)$.

- Serum miR-375-3p expression levels in early CRC patients (stage I/II) were higher than serum miR-375-3p levels in patients with advanced CRC (stage III/IV). 
Financial \& competing interests disclosure

This study was supported by Basic Research Project of Peking University Shenzhen Hospital (JCYJ2017001, JCYJ2017004, JCYJ2017005, JCYJ2017006, JCYJ2017007 and JCYJ2017012), Clinical Research Project of Peking University Shenzhen Hospital (BCYJ2017001), Science and Technology Development Fund Project of Shenzhen (no. JCYJ20180507183102747) and Clinical Research Project of Shenzhen Health Commission (no. SZLY2018023). The authors have no other relevant affiliations or financial involvement with any organization or entity with a financial interest in or financial conflict with the subject matter or materials discussed in the manuscript apart from those disclosed.

No writing assistance was utilized in the production of this manuscript.

\section{Ethical conduct of research}

The authors state that this study was reviewed and approved by the Ethics Committee of Shenzhen Hospital, Peking University. In addition, for investigations involving human subjects, informed consent has been obtained from the participants involved. We collected serum samples from 135 histopathologically confirmed colorectal adenocarcinoma (CRC) patients and 135 normal controls (NCs) from January 2016 to May 2019 at Shenzhen Hospital, Peking University (Shenzhen, China).

\section{Data sharing statement}

The authors state that this manuscript reports original results of a clinical trial.

\section{References}

Papers of special note have been highlighted as: • of interest

1. Siegel RL, Miller KD, Jemal A. Cancer statistics, 2019. CA Cancer J. Clin. 69(1), 7-34 (2019).

2. Hashiguchi Y, Muro K, Saito Y et al. Japanese Society for Cancer of the Colon and Rectum (JSCCR) guidelines 2019 for the treatment of colorectal cancer. Int.J. Clin. Oncol. doi:10.1007/s10147-019-01485-z 2019) (Epub ahead of print).

3. Baxter NN, Goldwasser MA, Paszat LF, Saskin R, Urbach DR, Rabeneck L. Association of colonoscopy and death from colorectal cancer. Ann. Intern. Med. 150(1), 1-8 (2009).

4. Malila N, Oivanen T, Malminiemi O, Hakama M. Test, episode, and programme sensitivities of screening for colorectal cancer as a public health policy in Finland: experimental design. BMJ337, a2261-a2261 (2008).

5. Hayes J, Peruzzi PP, Lawler S. MicroRNAs in cancer: biomarkers, functions and therapy. Trends Mol. Med. 20(8), 460-469 (2014).

6. Singh R, Ramasubramanian B, Kanji S, Chakraborty AR, Haque SJ, Chakravarti A. Circulating microRNAs in cancer: hope or hype? Cancer Lett. 381(1), 113-121 (2016).

- A comprehensive review about the feasibility and value of circulating miRNAs as cancer biomarkers.

7. Sohel MMH. Circulating microRNAs as biomarkers in cancer diagnosis. Life Sci. 248, 117473 (2020).

- A comprehensive review about the feasibility and value of circulating miRNAs as cancer biomarkers.

8. Nakamura K, Sawada K, Yoshimura A, Kinose Y, Nakatsuka E, Kimura T. Clinical relevance of circulating cell-free microRNAs in ovarian cancer. Mol. Cancer 15(1), 48 (2016).

9. Kirschner MB, Kao SC, Edelman JJ et al. Haemolysis during sample preparation alters microRNA content of plasma. PLoS ONE 6(9), e24145 (2011).

10. Livak KJ, Schmittgen TD. Analysis of relative gene expression data using real-time quantitative PCR and the 2(-Delta Delta C(T)) method. Methods 25(4), 402-408 (2001).

11. Gopalan V, Smith RA, Lam AK. Downregulation of microRNA-498 in colorectal cancers and its cellular effects. Exp. Cell Res. 330(2), 423-428 (2015).

12. Liu S, Gao G, Yan D et al. Effects of miR-145-5p through NRAS on the cell proliferation, apoptosis, migration, and invasion in melanoma by inhibiting MAPK and PI3K/AKT pathways. Cancer Med. 6(4), 819-833 (2017).

13. Zhang H, Jiang M, Liu Q, Han Z, Zhao Y, Ji S. miR-145-5p inhibits the proliferation and migration of bladder cancer cells by targeting TAGLN2. Oncol. Lett. 16(5), 6355-6360 (2018).

14. Mei LL, Wang WJ, Qiu YT, Xie XF, Bai J, Shi ZZ. miR-145-5p suppresses tumor cell migration, invasion and epithelial to mesenchymal transition by regulating the Sp1/NF-kappaB signaling pathway in esophageal squamous cell carcinoma. Int. J.Mol. Sci. 18(9), 1833 (2017).

15. Niu Y, Zhang J, Tong Y, Li J, Liu B. miR-145-5p restrained cell growth, invasion, migration and tumorigenesis via modulating RHBDD1 in colorectal cancer via the EGFR-associated signaling pathway. Int. J. Biochem. Cell Biol. 117, 105641 (2019).

- Previous expression profile of miR-203a-3p, miR-145-5p, miR-375-3p and miR-200c-3p in CRC tissue.

16. Cui F, Wang S, Lao I et al. miR-375 inhibits the invasion and metastasis of colorectal cancer via targeting SP1 and regulating EMT-associated genes. Oncol. Rep. 36(1), 487-493 (2016). 
- Previous expression profile of miR-203a-3p, miR-145-5p, miR-375-3p and miR-200c-3p in CRC tissue.

17. Mussnich P, Rosa R, Bianco R, Fusco A, D'angelo D. MiR-199a-5p and miR-375 affect colon cancer cell sensitivity to cetuximab by targeting PHLPP1. Expert Opin. Ther. Targets 19(8), 1017-1026 (2015).

18. Lu YX, Yuan L, Xue XL et al. Regulation of colorectal carcinoma stemness, growth, and metastasis by an miR-200c-Sox2-negative feedback loop mechanism. Clin. Cancer Res. 20(10), 2631-2642 (2014).

- Previous expression profile of miR-203a-3p, miR-145-5p, miR-375-3p and miR-200c-3p in CRC tissue.

19. Srivastava A, Moxley K, Ruskin R, Dhanasekaran DN, Zhao YD, Ramesh R. A non-invasive liquid biopsy screening of urine-derived exosomes for miRNAs as biomarkers in endometrial cancer patients. AAPS J. 20(5), 82 (2018).

20. Chen L, Gao H, Liang J et al. miR-203a-3p promotes colorectal cancer proliferation and migration by targeting PDE4D. Am. J. Cancer Res. 8(12), 2387-2401 (2018).

- Previous expression profile of miR-203a-3p, miR-145-5p, miR-375-3p and miR-200c-3p in CRC tissue.

21. Qian Z, Gong L, Mou Y, Han Y, Zheng S. MicroRNA203a3p is a candidate tumor suppressor that targets thrombospondin 2 in colorectal carcinoma. Oncol. Rep. 42(5), 1825-1832 (2019).

22. Wang Z, Zhao Z, Yang Y et al. MiR-99b-5p and miR-203a-3p function as tumor suppressors by targeting IGF-1R in gastric cancer. Sci. Rep. 8(1), 10119 (2018).

23. Jiang $\mathrm{N}$, Jiang $\mathrm{X}$, Chen $\mathrm{Z}$ et al. MiR-203a-3p suppresses cell proliferation and metastasis through inhibiting LASP1 in nasopharyngeal carcinoma. J. Exp. Clin. Cancer Res. 36(1), 138 (2017).

24. Chen QF, Kong JL, Zou SC et al. LncRNA LINC00342 regulated cell growth and metastasis in non-small cell lung cancer via targeting miR-203a-3p. Eur. Rev. Med. Pharmacol. Sci. 23(17), 7408-7418 (2019). 